\title{
Who should get surgical aortic valve replacement in the era of transcatheter aortic valve replacement? - a surgeon's perspective
}

\author{
Moritz C. Wyler von Ballmoos ${ }^{1,2}$, Michael J. Reardon ${ }^{1,2}$ \\ ${ }^{1}$ Department of Cardiovascular Surgery, Houston Methodist Hospital, Houston, TX, USA; ${ }^{2}$ Weill Cornell Medicine, New York, NY, USA \\ Correspondence to: Moritz C. Wyler von Ballmoos, MD, PhD, MPH. Department of Cardiovascular Surgery, Houston Methodist DeBakey Heart \& \\ Vascular Center, Houston, TX 77030, USA. Email: mcwylervonballmoos@houstonmethodist.org.
}

Submitted Mar 20, 2020. Accepted for publication May 01, 2020.

doi: $10.21037 /$ acs-2020-av-15

View this article at: http://dx.doi.org/10.21037/acs-2020-av-15

\section{The evolution of transcatheter aortic valve replacement trials}

Over the last decade, transcatheter aortic valve replacement (TAVR) has become a widely adopted technology (1). Two landmark trials established TAVR as a viable treatment option for non-operative candidates and those with extreme surgical risk, based on the Society of Thoracic Surgery Predicted Risk of Mortality (STS PROM) model for isolated AVR and as determined by the heart team (2-4). Concurrently, trials randomizing high-risk patients to TAVR or surgical aortic valve replacement (SAVR) demonstrated non-inferiority between the two (PARTNER 1A) or, in the case of the CoreValve High-Risk Trial, superiority of TAVR for all-cause mortality $(4,5)$. This led to the first FDA approval of TAVR in the United States. Subsequent intermediate-risk trials showed TAVR to be non-inferior in terms of all-cause mortality or disabling stroke $(6,7)$. These studies provided the first evidence that TAVR was noninferior to SAVR in terms of mortality, with comparable or lower stroke rate, less acute kidney injury, transfusions, atrial fibrillation, time in the ICU or hospital, and faster recovery of function and quality of life. However, this occurred at the cost of more vascular complications, higher permanent pacemaker implantation (PPI) and incidence of paravalvular leak (PVL). With similar mortality but differences with respect to other important outcomes, the intermediate-risk trials started to shed light on differences between the two treatment modalities, making the STS PROM but one criterion for selecting the appropriate AVR strategy. Finally, in 2019, the 1-year data from two trials comparing TAVR against SAVR in patients with a low risk of 30-day mortality for isolated SAVR, were presented and resulted in approval of TAVR by the FDA for the treatment or severe, symptomatic aortic stenosis (ssAS) in all STS PROM risk categories $(8,9)$. With this, the surgical risk profile has been removed permanently as the main deciding factor in justifying TAVR. In fact, these trials provide compelling evidence for the use of TAVR in the respective study sample. It is an understanding of what comprises the population tested, especially in low-risk patients, that is critical when applying these results to clinical practice.

\section{Generalizability of current data-who wasn't in the trials}

Two major groups were-per protocol or de facto-not included in the TAVR versus SAVR trials to date: patients with bicuspid aortic valves (BiAV) and younger patients $(<65$ years old) $(8,9)$. Several centers have reported their experience with TAVR in BiAV but these are descriptive or comparative studies based on observational data in few, highly selected patients. At this point, there is no data from a randomized study comparing TAVR and SAVR in patients with BiAV.

The other group mostly omitted from trials to date is young patients, specifically those below the age of 65 . Even in the low-risk TAVR trials, the mean age was above 70 , with less than $7 \%$ being under 65 years $(8,9)$. Meanwhile, the low-risk category comprised $80 \%$ of all patients undergoing SAVR in the most recent STS-Database analysis, and more than $50 \%$ of those patients were younger than 70 years (10).

In both the Evolut-LRT and PARTNER 3 trials, patients with unfavorable anatomic features were excluded 
from the trials $(8,9)$. Patients with complex coronary artery disease (CAD) (SYNTAX score $>32$ in PARTNER 3; $>22$ Evolut-LRT) were excluded as per protocol; but the average SYNTAX score of included patients in both trials was de facto synonymous with the absence of CAD. Overall, 14\% (Evolut-LRT) and 34\% (PARTNER 3) were screening failures $(8,9)$. Furthermore, screening committees composed of highly experienced proceduralists scrutinized each patient considered for inclusion into a trial, probabilistically favoring the enrollment of those expected to have good outcomes. Other patients not included were never even considered due to unfavorable anatomy, co-morbidities, and other reasons. Nonetheless, low-risk trials were designed to screen all patients, as evidenced by the demographic characteristics.

\section{How to decide which strategy is best}

TAVR and SAVR are two complimentary treatment options. The right course of action in each patient is a critical decision-making process that is best made by an interdisciplinary heart team. The team must consider STS PROM, life expectancy, anatomy of the aortic valve and root, comorbidities, and patient preference.

The reported outcomes in TAVR trials have indeed been excellent. Yet, it is important to apply those findings to clinical practice appropriately. Apart from generalizabitlity issues, differences in operator and institutional experience could drastically affect the results achieved. Close monitoring of outcomes using national databases is paramount to ensure the success seen in trials is effectively replicated in routine clinical practice. The low-risk trials, and PARTNER 3 specifically, should also be understood as a comparison of treatment strategy when ssAS is the main pathology, rather than a head-to-head comparison of isolated AVR. A quarter of patients in PARTNER 3 receiving SAVR underwent additional procedures. If these procedures were warranted to improve morbidity and mortality, a similar proportion of patients in the TAVR arm would be expected to suffer the consequences of interventions later or not at all. The 10-year follow-up scheduled for both low-risk trials is critical in addressing such questions.

Presently, we have limited data on how well TAVR performs as a treatment strategy in young patients, those with BiAV disease, significant $\mathrm{CAD}$, or moderate-severe pathologies affecting other valves. Predominantly, these patients are currently still best served with SAVR, a well- characterized treatment strategy even for these subgroups. We continue to learn about the long-term impact of PVL, and mortality related to PPI. Subclinical valve thrombosis and its effect on long-term mortality, stroke risk or small vessel disease (SVD) remain another area of controversy. TAVR valves are exposed to significant forces when loaded onto delivery systems, during post-dilation and possibly with non-nominal and eccentric expansion of the valve; how this affects durability is unclear. The morbidity and mortality of SAVR following TAVR valves has not been sufficiently characterized either. This question will grow in importance as TAVR valves appear to be subject to endocarditis and SVD at approximately the same rate as surgically implanted valves. Treatment of failed TAVR valves with a valve-invalve strategy also remains controversial. The issues of early and late coronary obstruction, patient-prosthesis mismatch and associated mortality dampen the enthusiasm for this approach, certainly in young and low-risk patients where redo-SAVR has excellent outcomes. Younger patients need a life-time strategy to manage aortic stenosis as they may outlive the durability of a first or second bioprosthesis. The most appropriate treatment sequence in that setting remains entirely unknown.

These unknowns must be honored in the process of counselling patients and the best approach in clinical practice remains transparency, especially when it comes to acknowledging the missing evidence.

\section{Acknowledgments}

Funding: None.

\section{Footnote}

Conflicts of Interest: MJR reported consulting work for Medtronic, Boston Scientific, and Abbott Medical. MCW von Ballmoos reported consulting work for Boston Scientific, and LivaNova.

Open Access Statement: This is an Open Access article distributed in accordance with the Creative Commons Attribution-NonCommercial-NoDerivs 4.0 International License (CC BY-NC-ND 4.0), which permits the noncommercial replication and distribution of the article with the strict proviso that no changes or edits are made and the original work is properly cited (including links to both the formal publication through the relevant DOI and the license). See: https://creativecommons.org/licenses/by-nc-nd/4.0/. 


\section{References}

1. Wyler von Ballmoos MC, Barker CM, Reardon MJ. When to choose surgery in the era of transcatheter aortic valve replacement. Interv Cardiol Clin 2018;7:425-33.

2. Popma JJ, Adams DH, Reardon MJ, et al. Transcatheter aortic valve replacement using a self-expanding bioprosthesis in patients with severe aortic stenosis at extreme risk for surgery. J Am Coll Cardiol 2014;63:1972-81.

3. Leon MB, Smith CR, Mack M, et al. Transcatheter aortic-valve implantation for aortic stenosis in patients who cannot undergo surgery. N Engl J Med 2010;363:1597-607.

4. Smith CR, Leon MB, Mack MJ, et al. Transcatheter versus surgical aortic-valve replacement in high-risk patients. $\mathrm{N}$ Engl J Med 2011;364:2187-98.

5. Adams DH, Popma JJ, Reardon MJ, et al. Transcatheter

Cite this article as: Wyler von Ballmoos MC, Reardon MJ. Who should get surgical aortic valve replacement in the era of transcatheter aortic valve replacement?-a surgeon's perspective. Ann Cardiothorac Surg 2020;9(6):487-489. doi: 10.21037/acs-2020-av-15 aortic-valve replacement with a self-expanding prosthesis. N Engl J Med 2014;370:1790-8.

6. Leon MB, Smith CR, Mack MJ, et al. Transcatheter or surgical aortic-valve replacement in intermediate-risk patients. N Engl J Med 2016;374:1609-20.

7. Reardon MJ, Van Mieghem NM, Popma JJ, et al. Surgical or transcatheter aortic-valve replacement in intermediaterisk patients. N Engl J Med 2017;376:1321-31.

8. Mack MJ, Leon MB, Thourani VH, et al. Transcatheter aortic-valve replacement with a balloon-expandable valve in low-risk patients. N Engl J Med 2019;380:1695-705.

9. Popma JJ, Deeb GM, Yakubov SJ, et al. Transcatheter aortic-valve replacement with a self-expanding valve in low-risk patients. N Engl J Med 2019;380:1706-15.

10. Thourani VH, Suri RM, Gunter RL, et al. Contemporary real-world outcomes of surgical aortic valve replacement in 141,905 low-risk, intermediate-risk, and high-risk patients. Ann Thorac Surg 2015;99:55-61. 